\title{
Spectroscopic Characteristics of Carbon Dots (C-Dots) Derived from Carbon Fibers and Conversion to Sulfur- Bridged C-Dots Nanosheets
}

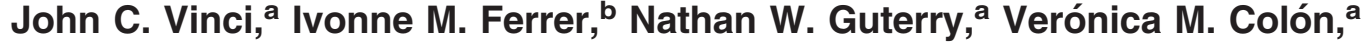 \\ Joel F. Destino, ${ }^{a}$ Frank V. Bright, ${ }^{a}$ Luis A. Colón ${ }^{a, *}$ \\ a State University of New York at Buffalo, Department of Chemistry, Natural Sciences Complex, Buffalo, NY 14260 USA \\ ${ }^{b}$ California Institute of Technology, Joint Center for Artificial Photosynthesis and Division of Chemistry and Chemical Engineering, \\ Pasadena, CA 91125 USA
}

\begin{abstract}
We synthesized sub-10 nm carbon nanoparticles (CNPs) consistent with photoluminescent carbon dots (C-dots) from carbon fiber starting material. The production of different C-dots fractions was monitored over seven days. During the course of the reaction, one fraction of C-dots species with relatively high photoluminescence was short-lived, emerging during the first hour of reaction but disappearing after one day of reaction. Isolation of this species during the first hour of the reaction was crucial to obtaining higherluminescent $\mathrm{C}$-dots species. When the reaction proceeded for one week, the appearance of larger nanostructures was observed over time, with lateral dimensions approaching $200 \mathrm{~nm}$. The experimental evidence suggests that these larger species are formed from small C-dot nanoparticles bridged together by sulfur-based moieties between the $\mathrm{C}$-dot edge groups, as if the $\mathrm{C}$-dots polymerized by cross-linking the edge groups through sulfur bridges. Their size can be tailored by controlling the reaction time. Our results highlight the variety of CNP products, from sub-10 nm C-dots to $\sim 200 \mathrm{~nm}$ sulfur-containing carbon nanostructures, that can be produced over time during the oxidation reaction of the graphenic starting material. Our work provides a clear understanding of when to stop the oxidation reaction during the top-down production of $\mathrm{C}$ dots to obtain highly photoluminescent species or a target average particle size.
\end{abstract}

Index Headings: Carbon dots; C-dots; Carbon nanoparticles; Photoluminescence nanoparticles; Carbon nanomaterials; Graphene oxide; Carbon nanosheets.

\section{INTRODUCTION}

A variety of bulk carbon-based starting materials such as soot, ${ }^{1-3}$ graphite nanofibers (GNFs), ${ }^{4,5}$ activated carbon, ${ }^{6}$ carbon nanotubes, ${ }^{7}$ graphene, ${ }^{8}$ and carbon fibers (CFs), ${ }^{9,10}$ have been used as precursors to perform top-down syntheses of carbon nanoparticles (CNPs). Species with relatively larger lateral dimensions such as graphene oxide nanosheets have been reported as products. ${ }^{5}$ However, smaller CNPs such as graphene quantum dots (GQDs) ${ }^{9}$ and carbon dots (C-dots) ${ }^{4}$ are often reported, which are similar species that generally differ in their height profiles as determined using atomic force microscopy (AFM). ${ }^{11}$ In addition, they may also have structural variety that can be determined using spectroscopic techniques such as Raman spectroscopy,

Received 29 September 2014; accepted 28 February 2015.

* Author to whom correspondence should be sent. E-mail: lacolon@ buffalo.edu.

DOI: $10.1366 / 14-07749$ in which significant differences can be found between amorphous C-dots ${ }^{12}$ and GQDs. ${ }^{13}$ Photoluminescent GQDs and C-dots are touted for their properties, such as small sizes, intrinsic and stable photoluminescence, biocompatibility, and ease of surface derivitization ${ }^{11}$ while showing promise for applications such as chromatographic stationary phases, ${ }^{14}$ drug-delivery vehicles, ${ }^{15}$ photothermal therapies, ${ }^{16}$ photocatalysis, ${ }^{17}$ bioimaging, ${ }^{4}$ and chemical sensing. ${ }^{18}$ Graphene oxide nanosheets are also cited for use in similar applications, including intracellular drug delivery and cellular imaging; ${ }^{19}$ in addition, graphene oxide can be chemically converted to graphene, ${ }^{20}$ which has a host of exciting applications and fascinating properties that have been at the center of research and innovation in recent years. ${ }^{21}$

The synthetic process to produce C-dots from bulk carbon starting materials involves the simultaneous etching (i.e., breaking of $\mathrm{C}-\mathrm{C}$ bonds) and oxidation of the bulk carbon starting material under acidic conditions; this produces species with hydrophilic moieties at the surfaces, making them water soluble. Typically, reaction conditions may involve temperatures around $80-160{ }^{\circ} \mathrm{C}$ and reaction times on the order of a few hours to a day. Previous studies of nanofiber-derived graphene oxide nanocolloids indicated that sheets are etched down from the nanofibers to sizes with average diameters of $46 \mathrm{~nm}$ for a $2 \mathrm{~h}$ reaction time and $18 \mathrm{~nm}$ for a $12 \mathrm{~h}$ reaction time. ${ }^{5} \mathrm{~A}$ variety of properties have been reported for CFderived GQDs synthesized at different reaction temperatures and a constant reaction time of one day. ${ }^{9}$ Carbon dots have also been synthesized from CFs by electrochemical etching; ${ }^{22}$ other reports of CF-derived CNPs use single-temperature and reaction-time conditions. ${ }^{10}$

Although there is a plethora of literature on various reaction conditions at different temperatures and oxidizing conditions using nitric acid only, ${ }^{3}$ nitric acidsulfuric acid mixtures, ${ }^{4}$ and modified Hummers' methods, ${ }^{5}$ there is a distinct lack of fundamental studies and understanding of the reaction dynamics and the species produced at various time points. Furthermore, a detailed study of intact CFs treated under a mixture of nitric and sulfuric acids has previously revealed that oxygen-, nitrogen-, and sulfur-containing groups varied discontinuously over time on the CF surface, especially in the early stages of reaction. ${ }^{23}$ The authors of this study $^{23}$ did not aim to study any nanomaterial production, but they found that acid attack occurred first on the CF surface, which established sites for subsequent 
oxidation. These results indicate that the etching of CNPs from CFs may be a dynamic process that warrants detailed study on its own.

One question we wanted to answer was: Can the same or similar CNP species be produced after the optimization of the reaction conditions from graphitic-based starting materials of vastly different dimensions $(80 \mathrm{~nm}$ versus $11 \mu \mathrm{m}$ diameter carbonaceous fibers)? Although this could seem trivial, there is no such direct evaluation in the literature. There are reports on using both CFs and GNFs to produces C-dots. ${ }^{4,5,9,10}$ Considering their costs (GNFs cost $\sim \$ 50.00 / \mathrm{g}$ and CFs $\sim \$ 0.10 / \mathrm{g}$ ), it is important to understand whether both can be used to generate the same species. We also wanted to understand how CNP species change over an extended period of time in the reaction vessel. That is, are they etched away into molecular precursors or does the nanomaterial somehow stabilize at favored products, or do the CNPs continue to evolve into new nanomaterial species as a function of time? Although it is useful to start with reported conditions to rapidly synthesize carbon nanomaterial, we contend it is of immediate importance to perform detailed studies of CNP products at various time points in the reaction to obtain the most desirable photoluminescent products and gain a fundamental understanding of the CNP production process.

In this study, we prepared C-dots using CFs as the graphenic carbon precursor and followed the reaction using anion-exchange high-performance liquid chromatography (AE-HPLC). The high-resolution separation of CNPs facilitated the study of the different species produced, allowing us to obtain unique and crucial information rapidly, on-line $e^{1,2,4}$ as well as off-line, ${ }^{1,4,24}$ after their fractionation. We provide evidence indicating that the first hour of the reaction is crucial to isolating unique photoluminescent C-dots species in solution. Further, by using a variety of techniques and extending the reaction time of the CFs out to seven days, we can see that clear day-to-day differences exist for the produced CNPs, with a trend suggesting that the C-dots "polymerize" to form larger nanostructures with covalent sulfur-based cross-links between the C-dot edge groups.

\section{MATERIALS AND METHODS}

Chemicals. Sulfuric acid (95-98\%) and nitric acid $(65 \%)$ (EM Science, Gibbstown, NJ) were used in the hydrothermal reaction and later neutralized with ammonium carbonate (J. T. Baker, Phillipsburg, NJ). The CFs (Union Carbide, Danbury, CT) acquired were of the Thornel brand (type P-55s, 4K, lot no. UC-320). A technical data sheet with the properties and characteristics for the CFs is readily available at the Cytec Industries website. ${ }^{25}$ Deionized water was collected from an EasyPure II system (Barnstead International) fed with a deionized water tap to produce $18.2 \mathrm{M} \Omega \cdot \mathrm{cm}$ deionized and filtered $(0.2 \mu \mathrm{m})$ water.

Synthesis of Carbon Nanoparticles. The carbon nanomaterial from the CFs was synthesized by placing $300 \mathrm{mg}$ of CFs and $60 \mathrm{~mL}$ sulfuric acid $\left(\mathrm{H}_{2} \mathrm{SO}_{4}\right)$ and $20 \mathrm{~mL}$ nitric acid $\left(\mathrm{HNO}_{3}\right)$ into a three-neck round-bottom flask fitted with a reflux condenser in the middle and the two additional ports capped. The reaction mixture was bath sonicated for $1 \mathrm{~h}$ prior to heating at $120^{\circ} \mathrm{C}$. A $1 \mathrm{~mL}$ aliquot of the reaction mixture was removed at each of the following time points: $1 \mathrm{~h}, 1$ day, 2 days, 3 days, 4 days, 5 days, 6 days, and 7 days. Ammonium carbonate was used to neutralize the samples. After neutralization, the samples were centrifuged for $10 \mathrm{~min}$ at $10000 \mathrm{rpm}$ using a mini-centrifuge (Corning/Costar, Cambridge, MA) and filtered through a $450 \mathrm{~nm}$ syringe filter. The filtered supernatant was dialyzed against water in a $1 \mathrm{kDa}$ highgrade regenerated cellulose tubular membrane (Membrane Filtration Products, Seguin, TX) to remove small species and salts. This produced CNPs for the different times in the range of $120-180 \mathrm{mg}$, corresponding to yields of $40-60 \%$. Control CNP syntheses were performed in which the CFs were exposed to the same conditions but using nitric acid only and sulfuric acid only. An amount of $80 \mathrm{~mL}$ of the acid was added for each control reaction. The post-synthetic treatment was performed in the same manner to obtain CNPs.

Spectroscopy. Raman spectroscopy was performed using a spectrophotometer (Horiba Jobin-Yvon, Edison, $\mathrm{NJ}$ ) equipped with a coupled-charged device (CCD) camera. The RTD exposure time was set to $1 \mathrm{~s}$, the accumulation exposure time was $1000 \mathrm{~s}$, and the number of accumulations was three. The $514.5 \mathrm{~nm}$ laser excitation line from an argon ion laser $(\leq 5 \mathrm{~mW})$ was employed. Fourier transform infrared (FT-IR) spectroscopy was carried out on a Hyperion 3000 IR microscope $\left(4 \mathrm{~cm}^{-1}\right.$ resolution, 64 scans, $15 \times$ objective; Bruker Optics, Billerica, MA) and Vertex 70 bench. Samples were analyzed in transmission mode. Atmospheric water and carbon dioxide $\left(\mathrm{CO}_{2}\right)$ were corrected, and baseline subtracted data were used. Measured spectra were referenced with respect to a blank area of the silicon wafer substrate used for measurement. X-ray photoelectron spectroscopy (XPS) measurements were performed under high vacuum conditions using a PHI 5000 VersaProbe XPS spectrophotometer with monochromatic AI $\mathrm{K} \alpha$ radiation (1486.6 eV) equipped with a hemispherical energy analyzer (Physical Electronics, Chanhassen, $\mathrm{MN})$. Samples were analyzed using a pass energy of $23.5 \mathrm{eV}$ and an increment of $0.1 \mathrm{eV}$ for high-resolution scans and a pass energy of $117.4 \mathrm{eV}$ and an increment of $1.0 \mathrm{eV}$ for survey scans. Prior to peak fitting, the spectra were baseline corrected using the PHI Multipak software (ver. 9.2.0.5). The high-resolution, fitted spectra were adjusted to normalize the $\mathrm{C}-\mathrm{C}$ peak to $284.6 \mathrm{eV}$. For relative elemental analysis, high-resolution $\mathrm{C} 1 \mathrm{~s}, \mathrm{O} 1 \mathrm{~s}$, N1s, and S2p scans were used and analyzed using the PHI Multipak software. For Raman, FT-IR, and XPS measurements, the samples were drop-cast onto a silicon wafer and dried prior to analysis. A Cary Eclipse fluorimeter (Agilent/Varian, Santa Clara, CA) was used for photoluminescence measurements, and ultravioletvisible (UV-Vis) spectroscopy was performed using a 8453 UV-visible absorption spectrophotometer (Agilent).

Separations. We used AE-HPLC to separate and analyze the CNPs using a IonPac AS12A analytical column $(4.0 \mathrm{~mm}$ inner diameter $\times 200 \mathrm{~mm}$ length; Dionex, Sunnyvale, CA) on an Agilent 1100 HPLC system (Agilent Technologies, Santa Clara, CA). Detection was achieved on-line using UV absorbance at $250 \mathrm{~nm}$ and laser- 
induced photoluminescence (LIP) detection. For LIP, the excitation source consisted of the $325 \mathrm{~nm}$ line of a helium cadmium ( $\mathrm{HeCd}$ ) laser (series 56; Omnichrome, Chino, CA). The emitted light was passed through a $350 \mathrm{~nm}$ longpass filter before reaching a photomultiplier tube detector. The separation conditions were as follows: flow rate, $1 \mathrm{~mL} / \mathrm{min}$; mobile phase gradient from 100 to $300 \mathrm{mM}$ ammonium carbonate ( $\mathrm{pH}$ 9.0) over $15 \mathrm{~min}$, held for $20 \mathrm{~min}$, and then flushed with $1.00 \mathrm{M}$ ammonium carbonate. For fraction collection, a semi-preparative column was used with dimensions of $9.0 \mathrm{~mm}$ (inner diameter) $\times 250 \mathrm{~mm}$ (length) with a flow rate of $5 \mathrm{~mL} / \mathrm{min}$, using the same separation conditions used with the analytical column. Capillary electrophoresis (CE) was performed on a P/ACE MDQ system equipped with a UV detection unit (Beckman Coulter, Brea, CA). The 32 Karat software (MDQ) was used for system operation and data collection. Electrophoretic separations were performed using a bare fused-silica capillary (Polymicro Technologies, Phoenix, AZ) with dimensions $50 \mu \mathrm{m}$ internal diameter, $60 \mathrm{~cm}$ total length, and $50 \mathrm{~cm}$ effective length. The applied voltage was $25 \mathrm{kV}$. The run buffer consisted of $100 \mathrm{mM}$ glycine (J.T. Baker, Phillipsburg, NJ) at pH 9.9. The data were collected at $4 \mathrm{~Hz}$ from the UV detector set at $214 \mathrm{~nm}$. The capillary temperature was maintained at $25{ }^{\circ} \mathrm{C}$. Sample injection was performed by applying 0.5 psi for $8 \mathrm{~s}$.

Electron Microscopy and Atomic Force Microscopy. Transmission electron microscopy (TEM) measurements were performed on a JEM 2010 (JEOL, Peabody, MA) operated at $200 \mathrm{kV}$. Samples were drop-cast on an ultrathin carbon film on holey carbon (from Ted Pella, Redding, CA). We carried out AFM using a SmartSPM1000-2 (AIST-NT, Novato, CA) with a Si probe $(k=5.3 \mathrm{~N} / \mathrm{m})$ in tapping mode.

\section{RESULTS AND DISCUSSION}

Synthesis and Fractionation of Carbon Dots. Carbon-fiber-derived CNPs were synthesized under various conditions using mixtures of nitric and sulfuric acids., ${ }^{9,10,26}$ After scouting various conditions, based on our previous work with GNF starting material, ${ }^{4,24}$ we settled on reaction conditions using a mixture of nitric and sulfuric acids at a constant temperature of $120{ }^{\circ} \mathrm{C}$. Our aim was to produce small, luminescent C-dots from CFs that may resemble luminescent nanomaterials obtained from less cost-effective GNF precursors ${ }^{4}$ under optimized conditions to maximize production.

We evaluated various reaction time points in the oxidation reaction. After $1 \mathrm{~h}$ of reaction under the acidic conditions, the CF starting material was completely etched into solution with no detectable solid CFs remaining in the reaction vessel (see Fig. S1A in the Supplemental Material). Interestingly, when performing the control experiments using nitric acid only or sulfuric acid only, we found that solid pieces of CF persisted even after two weeks of refluxing at $120^{\circ} \mathrm{C}$ (Figs. S1B-S1D). These results indicate that the combination of nitric and sulfuric acids is critical to the complete etching of CFs into water-soluble species. Using the nitric acid-sulfuric acid mixture, we found that, after $1 \mathrm{~h}$ of reaction, luminescent nanoparticle fractions were regenerated that had chro-

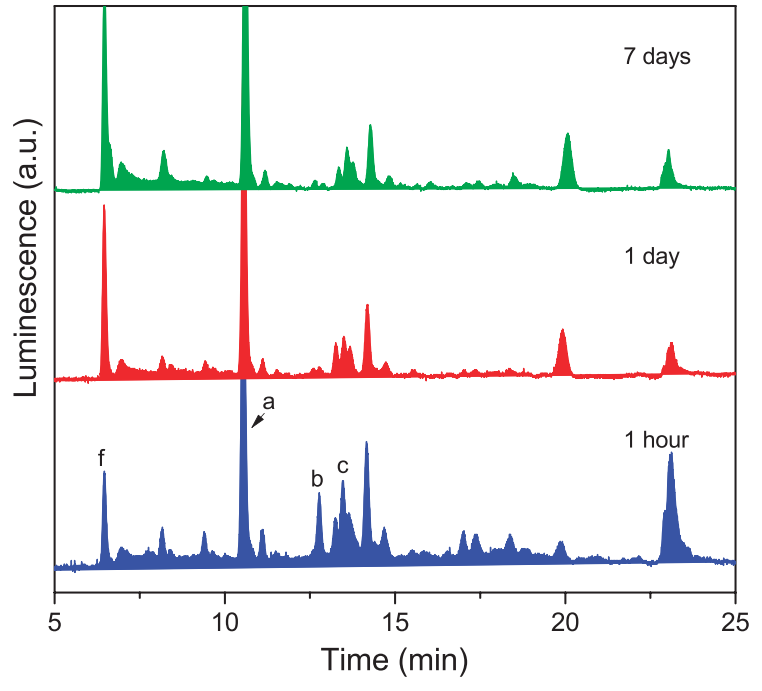

FIG. 1. The AE-HPLC chromatograms of CF-derived CNPs, synthesized after $1 \mathrm{~h}$, one day, and seven days of reaction time. Peak $\mathrm{b}$ was prominent in the $1 \mathrm{~h}$ reaction products but absent after one day of reaction.

matographic patterns and elution times similar to those obtained from GNFs. ${ }^{4}$ To investigate whether, indeed, different bulk carbon starting materials could be used to reproduce similar $\mathrm{C}$-dots species, the oxidation of $\mathrm{CFs}$ was followed for seven days using AE-HPLC (Fig. 1). The CF-derived fraction $\mathrm{b}$ in Fig. 1 showed nearly identical excitation-emission spectra (see Fig. $2 b$ ) and average size (TEM images shown in Fig. S2) to those of the GNFderived fraction having similar a retention time under identical conditions. ${ }^{4}$ Other fractions, for example, fractions $\mathrm{a}$ and $\mathrm{c}$ in Fig. 1, also showed strikingly similar properties to those derived from the GNFs (Fig. S3). We previously studied various HPLC-derived fractions of Cdots, and each fraction is known to have unique properties, such as photoluminescence, size, and surface functionality. ${ }^{1,4,24}$ The species a, b, and c in Fig. 1 differ in
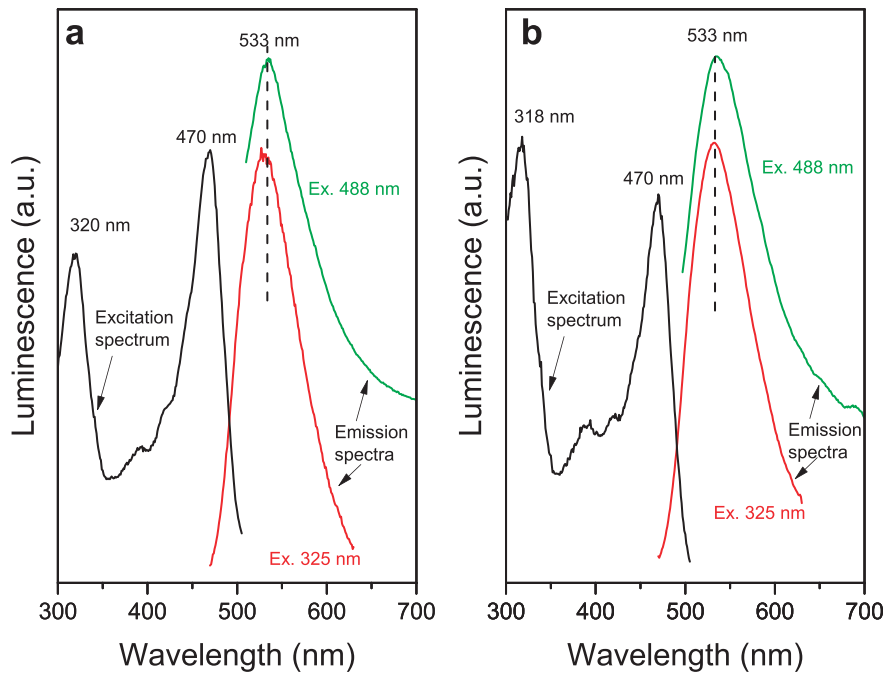

FIG. 2. Excitation and emission spectra (excitation $\lambda$ of 325 and $488 \mathrm{~nm}$ ) for fraction $b$ in both materials having identical retention times by AE-HPLC. (a) GNF-derived fraction. (b) CF-derived fraction. The emission spectrum for the $488 \mathrm{~nm}$ excitation has been offset in the $y$ axis for clarity in both panels. 

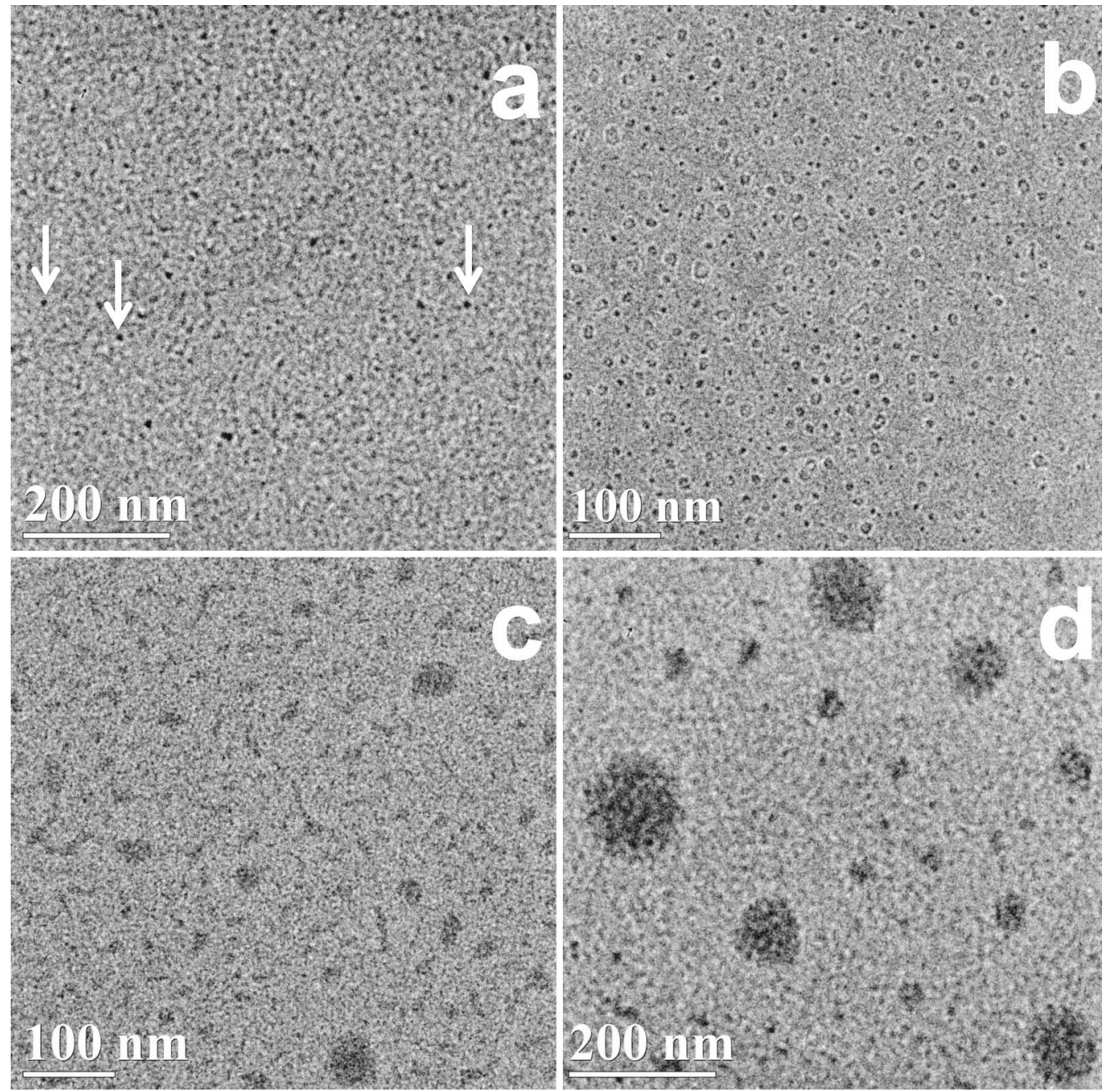

FIG. 3. The TEM images of the CF-derived CNPs synthesized. (a) After $1 \mathrm{~h}$; the arrows point to some of the small ( $\sim 7 \mathrm{~nm}) \mathrm{C}$-dots observed. (b) After 3 days. (c) After 5 days. (d) After 7 days.

charge, in that they elute at different times from the ion chromatographic column, and they also differ in size (species a is $10 \mathrm{~nm}, \mathrm{~b}$ is $7 \mathrm{~nm}$, and $\mathrm{c}$ is $14 \mathrm{~nm}$ ).

The CF-derived fraction $b$ in Fig. 1 was a transient species present after $1 \mathrm{~h}$ of reaction but was absent after one day of reaction and did not reappear through seven days of reaction. It is important to note that fraction $b$ corresponds to one of the species with higher quantum yields in the nanoparticle mixture (4\% quantum yield compared to $1 \%$ of the unseparated mixture), and without a detailed study of CNPs over time, such species could have been missed and eliminated entirely from the reaction products. To investigate the time-dependent CNP product distribution, we performed additional studies of the products after $1 \mathrm{~h}$ and after each day of reaction.

Analysis of the CF reaction mixture over time using TEM (see Fig. 3) revealed a clear trend of an increased particle size as a function of reaction time. Small CNPs, similar to what was isolated as AE-HPLC fraction b (Fig. S2), were found in the $1 \mathrm{~h} \mathrm{CF-derived} \mathrm{CNP} \mathrm{products} \mathrm{(Fig.}$ 3a). The lowest particle heights observed for the CNPs synthesized after $1 \mathrm{~h}$ of reaction were 1.5-3 nm, determined by AFM (Fig. S4A). Carbon nanoparticles with such a height profile have been ascribed in the literature to few-layer or multilayer graphene quantum dots $^{27}$ and C-dots. ${ }^{12}$ Using high-resolution TEM, we did not observe a defined crystalline lattice; therefore, we do not claim our CNPs are graphene quantum dots. After three days of reaction, there was a clear change of the sub-10 nm C-dots to a mixture of C-dots and larger species 10-20 nm in diameter (Fig. 3b). Even larger species were observed after five days of reaction (Fig. 3c), and the largest species, approaching $200 \mathrm{~nm}$ in diameter, were found after seven days of reaction. The large species produced after seven days of reaction appeared to be planar nanostructures, based on the contrast of the TEM image (Fig. 3d), although these species incorporated sulfur in their structure. Some small C-dots species could still be found after seven days of reaction alongside the larger disk-like carbon nanostructures. We confirmed using AFM that the height of the larger CNPs synthesized after seven days was 4-7 nm (Fig. S4B), which is indicative of a multilayer structure. The AFM images also highlighted the differences in particle dimensions between $1 \mathrm{~h}$ and 7 days of reaction, suggesting some sort of particle growth (i.e., 


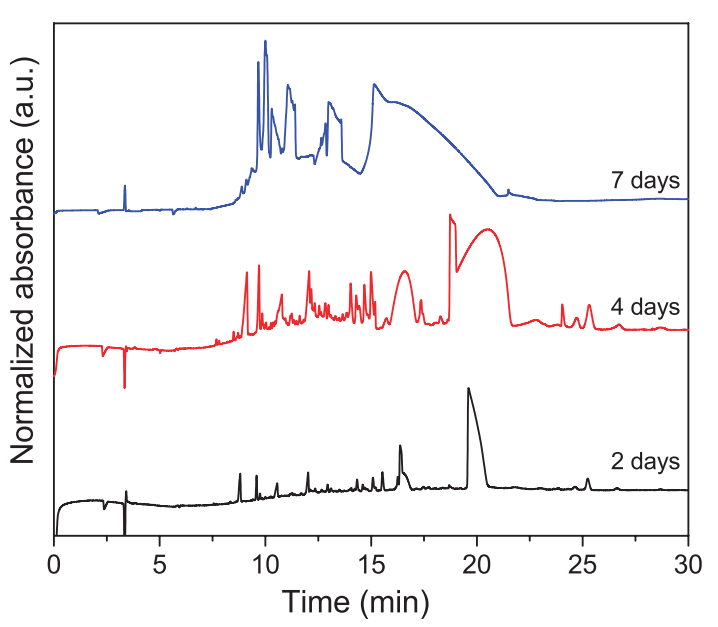

FIG. 4. Electropherograms of the CF-derived CNPs after two days, four days, and seven days of reaction.

polymerization) over time, with the larger particle sizes found after seven days of reaction (see Figs. 3d and S4).

The top-down CNP formation is considered an etching process resulting in smaller structures derived from the starting material; it was surprising to see particle growth on the three to seven day time scale. Most of the reported CNP syntheses have been performed on much shorter time scales, ${ }^{5,9,28}$ possibly missing this phenomenon. The results from AE-HPLC indicated some changes in the CNPs at different reaction times, but they were minimal compared to the vast differences seen using TEM. Separation using AE-HPLC is based on anionic surface interaction with the ion-exchange resin in the HPLC column. If the C-dots were linked together to form larger structures over time, they may have been too large to make it through the chromatographic column. In addition, the surface-charge properties of the larger nanoparticles may have also decreased significantly, and those nanoparticles of appropriate size may have little or no interaction with the retentive phase under the experimental conditions used. This is supported by the increase in the peak height of the chromatographic band corresponding to the least retained compound species (species $f$ in Fig. 1). To learn more about the species formed at longer reaction times, we turned to other techniques.

Capillary electrophoresis is a separation technique based on an analytes' charge/size ratio, and it can provide indications of various sizes of CNPs. ${ }^{29}$ The CE of the reaction mixtures at different reaction times showed that the charge/size ratio of the species changed over time (see Fig. 4). After two days, the electropherogram showed multiple CNP species, mostly defined by individual peaks. However, after four days of reaction, significant peak broadening was observed, which continued to broaden as the time progressed to seven days of reaction, as seen in Fig. 4. The broadening of electrophoretic peaks is indicative of a broadening of the CNP species charge/size distribution of the CNP, ${ }^{20}$ which we attribute to CNPs with larger sizes, while maintaining broad distributions of CNPs from sub-10 nm to near $200 \mathrm{~nm}$ after seven days of reaction.
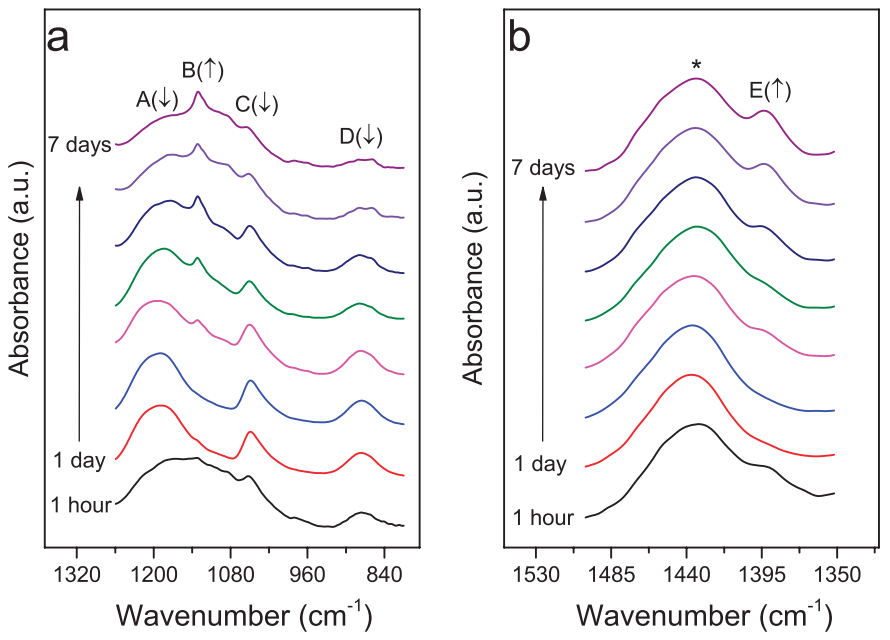

FIG. 5. Offset, normalized FT-IR spectra of the CF-derived CNPs after $1 \mathrm{~h}$ and from one to seven days of reaction time (by one day increments). (a) For the $1260-800 \mathrm{~cm}^{-1}$ region. (b) For the 1500$1350 \mathrm{~cm}^{-1}$ region. Region $\mathrm{A}(\downarrow)\left(\sim 1190 \mathrm{~cm}^{-1}\right)$, region $\mathrm{C}(\downarrow)\left(\sim 1050 \mathrm{~cm}^{-1}\right)$, and region $\mathrm{D}(\downarrow)\left(\sim 880 \mathrm{~cm}^{-1}\right)$ are assigned to sulfonic acids. Region $\mathrm{B}(\uparrow)$ $\left(\sim 1130 \mathrm{~cm}^{-1}\right)$ and region $\mathrm{E}(\uparrow)\left(\sim 1400 \mathrm{~cm}^{-1}\right)$ are assigned to covalent sulfonates. The up/down arrow for each region indicates increasing ( $\uparrow)$ or decreasing $(\downarrow)$ intensity over time.

Fourier Transform Infrared Spectroscopy. We used FT-IR spectroscopy to gain insight into the chemical composition of the time-dependent changes over the course of the evolution of C-dots to larger CNPs. The infrared (IR) spectra for the CNPs show a number of bands corresponding to sulfonic acids that decreased over time while the bands related to covalent sulfur bridges grew in magnitude (see Fig. 5). The timedependent FT-IR spectra show a loss and growth of certain functional groups. This phenomenon becomes more apparent after one day of reaction. The regions in the IR spectra corresponding to different functional groups are shown in Fig. 5a. The functional groups in region $A\left(\sim 1190 \mathrm{~cm}^{-1}\right)$ are assigned to sulfonic acid group $\left(\mathrm{SO}_{3}\right)$ asymmetrical stretching of sulfonic acid salts (some of the sulfonic acids converted to salts after neutralization), whereas region $C\left(\sim 1050 \mathrm{~cm}^{-1}\right)$ is assigned to the $\mathrm{SO}_{3}$ symmetrical stretching of sulfonic acid salts. ${ }^{30,31}$ Furthermore, region $\mathrm{D}\left(\sim 880 \mathrm{~cm}^{-1}\right)$ may be assigned to S-O stretching of sulfonic acids, ${ }^{31}$ although it may have some contribution from vinylidenes $^{32}$ because a peak near $880 \mathrm{~cm}^{-1}$ appears in the FT-IR spectra for both the $\mathrm{NO}_{3}$-only and $\mathrm{HSO}_{4}$-only control experiments (Fig. S5). Region B $\left(\sim 1130 \mathrm{~cm}^{-1}\right)$ in Fig. 5 shows the growth of the sulfonyl group $\left(\mathrm{SO}_{2}\right)$ symmetrical stretch of covalent sulfonates, ${ }^{30,33}$ concomitant with a decrease of the sulfonic acid functionality (regions $A$ and $C$ ). Further, a band grew over time around $1400 \mathrm{~cm}^{-1}$ (Fig. 5b, region E), which is assigned to the $\mathrm{SO}_{2}$ asymmetrical stretching of covalent sulfonates. ${ }^{30,32-34}$ The spectra in Fig. 5 were normalized to the band around $1435 \mathrm{~cm}^{-1}$ (identified by an asterisk), which is present in all spectra and can be assigned to $\mathrm{C}=\mathrm{C}$ bonding. ${ }^{31,35}$ Note that a peak near $1435 \mathrm{~cm}^{-1}$ appeared in the FT-IR spectra for both the $\mathrm{NO}_{3}$-only and $\mathrm{HSO}_{4}$-only control reactions (Fig. S5) indicating that it may be related to the carbon structure and not to sulfur- or nitrogen-containing functionalities. 

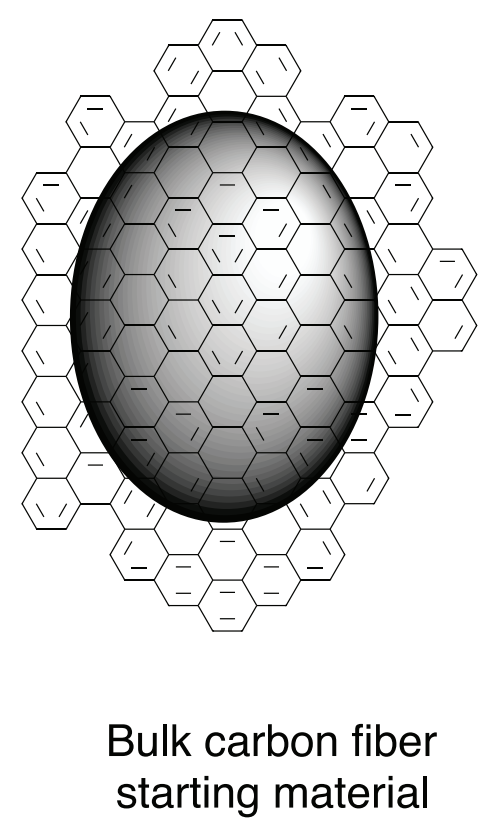
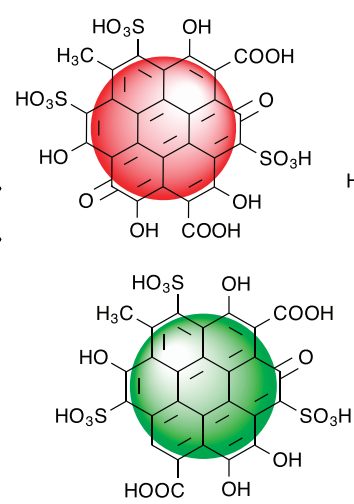

$\mathrm{HNO}_{3} / \mathrm{H}_{2} \mathrm{SO}_{4}$

1 Hour reaction
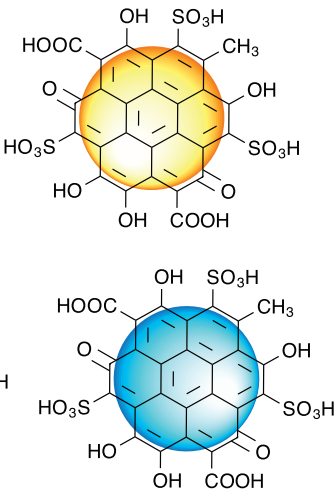

$\mathrm{HNO}_{3} / \mathrm{H}_{2} \mathrm{SO}_{4}$

7 Days reaction

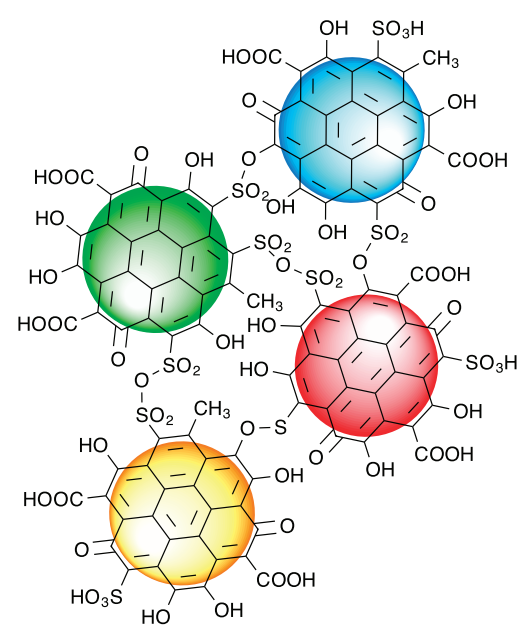

\section{Sulfur-based bridged C-dots}

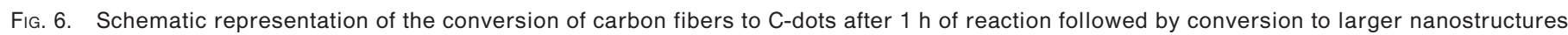
after dehydration reactions of sulfonic acids. Note that all functional groups were intentionally placed on the edges for simplicity.

The FT-IR spectra presented in Fig. 5 indicate that sulfonic acid residues on the CNPs are converted into covalent sulfonates over time. Covalent sulfonates could be formed through dehydration reactions with a sulfonic acid group and a hydroxyl group $(-\mathrm{OH})$ present on the CNPs. Interestingly, sulfur-based anhydrides also have $\mathrm{SO}_{2}$ symmetrical and asymmetrical stretching bands in the same region as the covalent sulfonates, ${ }^{30}$ and so anhydride-based covalent bridges arising from the dehydration of two sulfonic acid groups must not be discounted as a possibility. We note that sulfonic acids on Nafion membranes have been shown to condense into sulfonic acid anhydrides under acidic conditions. ${ }^{36}$ It is also possible to imagine the formation of sulfonic acid-carboxylic acid anhydrides. In any case, sulfonic acids appear to undergo dehydration to form covalent cross-links between nanoparticles, thus forming larger CNP structures as the reaction time proceeds on the day time scale. Note that non-sulfur-based functional groups present on the CNPs include alcohols, carbonyls, and carboxylates (Fig. S6). Other evidence for the loss of sulfonic acids over time is seen by monitoring the bands in the IR spectrum near 2475 and $2890 \mathrm{~cm}^{-1}$, region $\mathrm{F}$ and region $\mathrm{G}$, respectively, in Fig. S7, which can be assigned to sulfonic acids (-OH stretching); ${ }^{25,31}$ both decreased in intensity on the day time scale.

We cannot discard the possibility that the bridging that forms the larger nanostructures can also involve, for example, a carboxylate ester. In general, however, the FT-IR data strongly suggest that the C-dots produced contain the sulfonic acid functionality after one day of reaction, while covalent sulfonates (or anhydrides) grow systematically over the course of the seven days of reaction. The growth of covalent sulfur-based bridges by dehydration reactions of sulfonic acids is consistent with the formation of larger CNP phenomenon observed on the day time scale, which is schematically depicted in Fig. 6 . Note that, aside from the side-edge group stitching of the C-dots, condensation can also link C-dots across their basal planes, all in a polymerization-like fashion. The TEM images (Fig. 3) are indicative not just of larger particles connected through edge groups, but also of an increase in electron density contrast consistent with the increasing AFM height profiles (Fig. S4); this suggests that the condensation reactions are contributing to the connectivity of these structures to some degree in the $z$ direction, although sulfur bridging in the $x y$ direction predominates. We also point out that on the formation of the sulfur-containing nanostructures, we did not observe a measureable effect on the overall quantum yield of the bulk mixture, which remained at about $1 \%$ throughout the course of the reaction over seven days. However, the smaller C-dot nanoparticles (e.g., fraction b) disappeared as the sulfur-based moieties increase with reaction time.

Raman and X-ray Photoelectron Spectroscopies. To further understand the changes in structure and functionality of the CNPs over time, we also performed Raman spectroscopy and XPS. Raman spectroscopy indicated that after $1 \mathrm{~h}$ of synthesis, the $\mathrm{C}$-dots had a relatively high degree of graphitization, as indicated by the ratio of intensities of the D-band to G-band $\left(I_{\mathrm{D}} / /_{\mathrm{G}}\right)$, shown in Fig. 7 . The disorder D-band is related to defects in the graphite lattice (i.e., $\mathrm{sp}^{3}$ carbon), and the G-band is related to $\mathrm{sp}^{2}$ hybridized carbon networks. ${ }^{37}$ We found that after $1 \mathrm{~h}$ of synthesis, the $I_{D} / I_{G}$ ratio was $0.80 \pm 0.03$, indicating that there are some defects in the C-dots' $\mathrm{sp}^{2}$-carbon lattice arising from oxidation and hetero-groups on the surface. ${ }^{38}$ Such a ratio for the D-band to G-band is in the range that has been reported for high-quality CNPs. ${ }^{9,13}$ Over time, as oxidation continues and the $\mathrm{sp}^{2}$-carbon lattice is defected further, the $I_{D} / I_{G}$ ratio of the CNPs increases significantly $(p<0.05)$ to $0.94 \pm 0.02$ after one day of reaction, with a further increase $(p<0.05)$ to $1.01 \pm 0.04$ after seven days of reaction. We also point out that, although we observed the larger particles, the dimensions of the constituent 


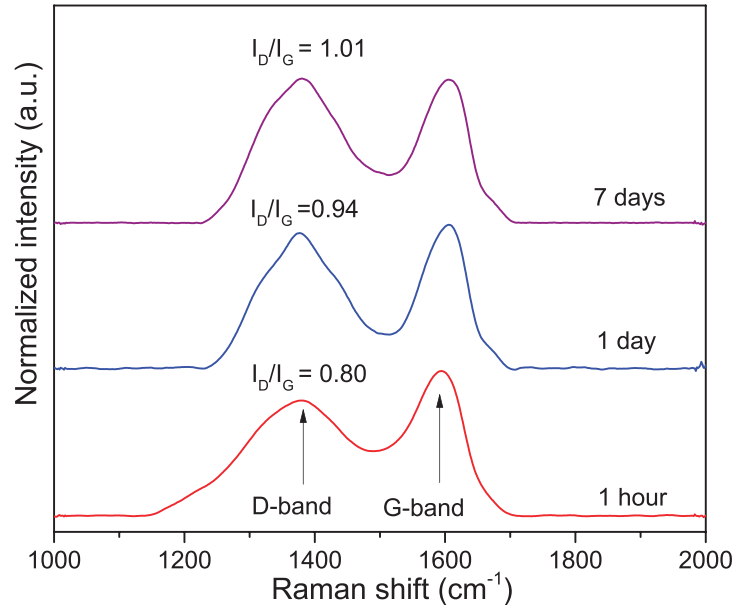

FIG. 7. Offset, normalized Raman spectra for CF-derived CNPs synthesized after $1 \mathrm{~h}$, one day, and seven days of reaction time. The D-band and G-bands are labeled, and the ratios of the intensities of the D-band to the G-band are provided for each spectrum.

fragments remained small, reflected in the D-band because the phonon coherence length is directly related to the Raman D-band. ${ }^{39}$

An XPS survey scan and a high-resolution scan (for carbon C1s) of the C-dots synthesized after $1 \mathrm{~h}$ of reaction are shown in Fig. 8. The scans indicate that a significant amount of oxygen is present on the carbonbased nanomaterial surface with some contributions from sulfur and nitrogen, too. We curve-fitted the highresolution $\mathrm{C} 1 \mathrm{~s}$ spectra by assuming the presence of $\mathrm{C}-\mathrm{C}-, \mathrm{C}-\mathrm{O}-, \mathrm{C}=\mathrm{O}-$, and $\mathrm{O}-\mathrm{C}=\mathrm{O}$-type bonding; although carbon bound to sulfur and nitrogen may also have some contributions in the same spectral region, they were disregarded because of the relatively low percentages of those species. A high-resolution, curve-fitted C1s spectrum for CNPs synthesized after seven days of reaction indicated similar types of bonding (Fig. S8). The different amount of carbon as $\mathrm{C}-\mathrm{C}, \mathrm{C}-\mathrm{O}, \mathrm{C}=\mathrm{O}$, and $\mathrm{O}-\mathrm{C}=\mathrm{O}$ bonding on the CNPs after $1 \mathrm{~h}$ and seven days of reactions are shown in Table I. Although the carbon percentages in Table I (panel A) seems to indicate a slight loss, the values for the amount of carbon as $\mathrm{C}-\mathrm{C}$ $(p=0.122)$ and as $\mathrm{C}-\mathrm{O}(p=0.060)$ are not significantly different for the two reaction times. There is, however, a notable increase of the higher oxidation-state functional group $\mathrm{O}-\mathrm{C}=\mathrm{O}(p=0.023)$, but the change in $\mathrm{C}=\mathrm{O}$ was not significant $(p=0.375)$. Table I (panel $B$ ) displays the XPS elemental composition using high-resolution scans of C1s, O1s, N1s, and S2p after $1 \mathrm{~h}$ and seven days of reaction. A relative loss of total carbon from 57 to $52 \%$
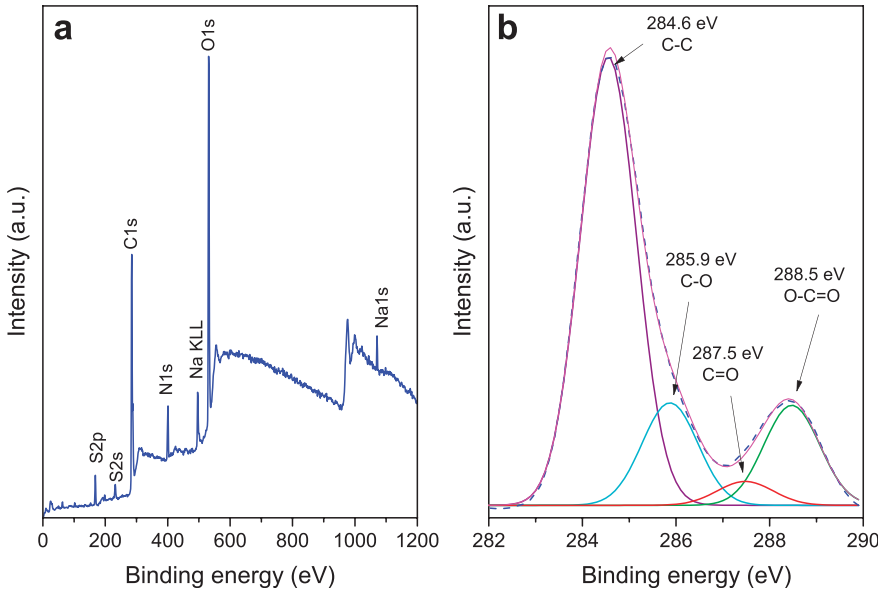

FIG. 8. Scans of the CF-derived CNPs after $1 \mathrm{~h}$ of synthesis. (a) The XPS survey scan. (b) High-resolution C1s scan. The dashed black line in (b) represents the measured spectrum, while the closely following continuous curve (magenta) represents the sum of the fitted peaks Individual spectra corresponding to the different oxidation states of carbon are indicated in (b).

$(p<0.001)$ can be noticed. Over the same time period, the percentages of oxygen, nitrogen, and sulfur all increased $(p<0.001)$ as some of the $\mathrm{sp}^{2}$-carbon lattice was transformed into $\mathrm{sp}^{3}$-carbon that was defective with hetero-groups. The increase in hetero-groups, however, was evidenced by electronic absorbance spectroscopy (Fig. S9). The UV-Vis peaks around $230 \mathrm{~nm}$ can be assigned to the $\pi-\pi^{*}$ transitions of $\mathrm{sp}^{2}$ carbon, ${ }^{40,41}$ indicating that graphitic carbon persisted through seven days of reaction, which we also confirmed using Raman spectroscopy. The shoulders from 250 to $400 \mathrm{~nm}$ may be assigned to $\mathrm{C}=\mathrm{O}$ groups $^{40,41}$ and other heterogroups, which can be clearly seen as increasing over the course of the reaction.

\section{CONCLUSION}

We have shown that certain thermodynamically favorable C-dots species with similar properties can be generated from two different bulk carbon precursors of vastly different dimensions (i.e., $11 \mu \mathrm{m}$ CFs and $80 \mathrm{~nm}$ GNFs). By means of AE-HPLC, we discovered that some CNP species with relatively high photoluminescence were transient and would have gone unperceived without our detailed study. Although we may expect further etching of the CNPs into smaller structures over time, we found that over the course of the reaction from one to seven days there is a clear nanostructure growth, producing CNPs near $200 \mathrm{~nm}$ in size after seven days of

TABLE I. Relative percentages of carbon-carbon and carbon-oxygen bonding and relative elemental composition for CF-derived CNPs synthesized.

\begin{tabular}{|c|c|c|c|c|c|c|c|c|}
\hline Reaction time & \multicolumn{4}{|c|}{ Carbon-carbon and carbon-oxygen bonding $(\%)^{a}$} & \multicolumn{4}{|c|}{ Elemental composition (\%) ${ }^{\mathrm{b}}$} \\
\hline $1 \mathrm{hr}$ & $68.3 \pm 0.5$ & $13.6 \pm 0.2$ & $3.1 \pm 0.4$ & $15.0 \pm 0.4$ & $57.1 \pm 0.3$ & $34.4 \pm 0.4$ & $2.4 \pm 0.1$ & $6.1 \pm 0.4$ \\
\hline
\end{tabular}

a Determined from peak fitting of C1s XPS spectra.

${ }^{b}$ Determined from high-resolution XPS scans. 
reaction. We attribute these to the reaction between individual C-dots that were bridged together through sulfur linkages from dehydration reactions of sulfonic acids, resulting in nanosheet-like structures. In this sense, bulk CFs are broken down to form C-dots (topdown) followed by the formation of larger nanoparticles from C-dots connected over time through their edge groups by sulfur-based bridges (bottom-up). The formation of the sulfur-bridged particles can be tuned to specific average diameters depending on the amount of time the reaction is allowed to proceed. Considering the recent interest in sub-10 $\mathrm{nm}$ photoluminescent CNPs and related materials, our results demonstrate that CFs are a viable starting material for synthesizing a range of CNPs that can be controlled by the reaction time. Significantly, careful control of reaction time and process monitoring is crucial to obtain highly luminescent C-dot species as well as to tailor the average particle size.

\section{ACKNOWLEDGMENTS}

We acknowledge the financial support for this study by the National Science Foundation, USA: Grants CHE 1058373, CHE 1048740 (CRIFMU), CHE-0848171, and CHE-1126301. The authors thank Dr. David Watson for use of the UV-Vis and fluorescence spectrophotometers, Dr. Sarbajit Banerjee for providing access to the Raman spectrophotometer, and Dr. Joseph A. Gardella for discussions on XPS.

\section{SUPPLEMENTAL MATERIAL}

All supplemental material mentioned in the text, which includes nine figures, can be found in the online version of the journal, at http://www. s-a-s.org.

1. 1. J.C. Vinci, L.A. Colon. "Fractionation of Carbon-Based Nanomaterials by Anion-Exchange HPLC". Anal. Chem. 2012. 84(2): 1178-1183.

2. J.S. Baker, L.A. Colon. "Influence of Buffer Composition on the Capillary Electrophoretic Separation of Carbon Nanoparticles". J. Chromatogr. A. 2009. 1216(52): 9048-9054.

3. H. Liu, T. Ye, C. Mao. "Fluorescent Carbon Nanoparticles Derived from Candle Soot". Angew. Chem. Int. Ed. 2007. 46(34): 6473-6475.

4. J.C. Vinci, I.M. Ferrer, S.J. Seedhouse, A.K. Bourdon, J.M. Reynard, B.A. Foster, F.V. Bright, L.A. Colon. "Hidden Properties of Carbon Dots Revealed After HPLC Fractionation". J. Phys. Chem. Lett. 2013. 4(2): 239-243.

5. J. Luo, L.J. Cote, V.C. Tung, A.T.L. Tan, P.E. Goins, J. Wu, J. Huang. "Graphene Oxide Nanocolloids". J. Am. Chem. Soc. 2010. 132(50): 17667-17669.

6. Y. Dong, N. Zhou, X. Lin, J. Lin, Y. Chi, G. Chen. "Extraction of Electrochemiluminescent Oxidized Carbon Quantum Dots from Activated Carbon". Chem. Mater. 2010. 22(21): 5895-5899.

7. D.V. Kosynkin, A.L. Higginbotham, A. Sinitskii, J.R. Lomeda, A. Dimiev, B.K. Price, J.M. Tour. "Longitudinal Unzipping of Carbon Nanotubes to Form Graphene Nanoribbons". Nature. 2009. 458(7240): 872-876.

8. D. Pan, J. Zhang, Z. Li, M. Wu. "Hydrothermal Route for Cutting Graphene Sheets into Blue-Luminescent Graphene Quantum Dots". Adv. Mater. 2010. 22(6): 734-738.

9. J. Peng, W. Gao, B.K. Gupta, Z. Liu, R. Romero-Aburto, L. Ge, L. Song, L.B. Alemany, X. Zhan, G. Gao, S.A. Vithayathil, B.A. Kaipparettu, A.A. Marti, T. Hayashi, J.-J. Zhu, P.M. Ajayan. "Graphene Quantum Dots Derived from Carbon Fibers". Nano Lett. 2012. 12(2): 844-849.

10. M. Xie, Y. Su, X. Lu, Y. Zhang, Z. Yang, Y. Zhang. "Blue and Green Photoluminescence Graphene Quantum Dots Synthesized from Carbon Fibers". Mater. Lett. 2013. 93: 161-164.

11. S.N. Baker, G.A. Baker. "Luminescent Carbon Nanodots: Emergent Nanolights". Angew. Chem. Int. Ed. 2010. 49(38): 6726-6744.

12. R. Liu, D. Wu, S. Liu, K. Koynov, W. Knoll, Q. Li. “An Aqueous Route to Multicolor Photoluminescent Carbon Dots Using Silica Spheres as Carriers". Angew. Chem. 2009. 121(25): 4668-4671.
13. H. Tetsuka, R. Asahi, A. Nagoya, K. Okamoto, I. Tajima, R. Ohta, A. Okamoto. "Optically Tunable Amino-Functionalized Graphene Quantum Dots". Adv. Mater. 2012. 24(39): 5333-5338.

14. Y. Li, L. Xu, T. Chen, X. Liu, Z. Xu, H. Zhang. "Carbon Nanoparticles from Corn Stalk Soot and Its Novel Application as Stationary Phase of Hydrophilic Interaction Chromatography and Per Aqueous Liquid Chromatography". Anal. Chim. Acta. 2012. 726: 102-108.

15. Q. Wang, X. Huang, Y. Long, X. Wang, H. Zhang, R. Zhu, L. Liang, P. Teng, H. Zheng. "Hollow Luminescent Carbon Dots for Drug Delivery”. Carbon. 2013. 59: 192-199.

16. J.T. Robinson, S.M. Tabakman, Y. Liang, H. Wang, H. Sanchez Casalongue, D. Vinh, H. Dai. "Ultrasmall Reduced Graphene Oxide with High Near-Infrared Absorbance for Photothermal Therapy". J. Am. Chem. Soc. 2011. 133(17): 6825-6831.

17. S. Zhuo, M. Shao, S.-T. Lee. "Upconversion and Downconversion Fluorescent Graphene Quantum Dots: Ultrasonic Preparation and Photocatalysis". ACS Nano. 2012. 6(2): 1059-1064.

18. J.-M. Liu, L.-p. Lin, X.-X. Wang, S.-Q. Lin, W.-L. Cai, L.-H. Zhang, Z.Y. Zheng. "Highly Selective and Sensitive Detection of $\mathrm{Cu}^{2+}$ with Lysine Enhancing Bovine Serum Albumin Modified-Carbon Dots Fluorescent Probe”. Analyst. 2012. 137(11): 2637-2642.

19. Q. Mu, G. Su, L. Li, B.O. Gilbertson, L.H. Yu, Q. Zhang, Y.-P. Sun, B. Yan. "Size-Dependent Cell Uptake of Protein-Coated Graphene Oxide Nanosheets". ACS Appl. Mater. Interfaces. 2012. 4(4): 22592266.

20. M.B. Müller, J.P. Quirino, P.N. Nesterenko, P.R. Haddad, S. Gambhir, D. Li, G.G. Wallace. "Capillary Zone Electrophoresis of Graphene Oxide and Chemically Converted Graphene”. J. Chromatogr. A. 2010. 1217(48): 7593-7597.

21. K.S. Novoselov, V.I. Fal'ko, L. Colombo, P.R. Gellert, M.G. Schwab, K. Kim. "A Roadmap for Graphene". Nature. 2012. 490(7419): 192200.

22. L. Bao, Z.-L. Zhang, Z.-Q. Tian, L. Zhang, C. Liu, Y. Lin, B. Qi, D.-W. Pang. "Electrochemical Tuning of Luminescent Carbon Nanodots: From Preparation to Luminescence Mechanism”. Adv. Mater. 2011. 23(48): 5801-5806.

23. G. Zhang, S. Sun, D. Yang, J.-P. Dodelet, E. Sacher. "The Surface Analytical Characterization of Carbon Fibers Functionalized by $\mathrm{H}_{2} \mathrm{SO}_{4} / \mathrm{HNO}_{3}$ Treatment". Carbon. 2008. 46(2): 196-205.

24. J.C. Vinci, L.A. Colón. "Surface Chemical Composition of Chromatographically Fractionated Graphite Nanofiber-Derived Carbon Dots". Microchem. J. 2013. 110: 660-664.

25. Cytec Industries. "Thornel ${ }^{\circledR}$ P-25 Pitch-Based Fiber, Technical Data Sheet". Tempe, AZ: Cytec, 2012. http://cytec.com/sites/default/files/ datasheets/THORNEL_P25_052112.pdf.

26. Y. Wang, Z. Iqbal, S. Mitra. "Rapidly Functionalized, WaterDispersed Carbon Nanotubes at High Concentration". J. Am. Chem. Soc. 2006. 128(1): 95-99.

27. L. Tang, R. Ji, X. Cao, J. Lin, H. Jiang, X. Li, K.S. Teng, C.M. Luk, S. Zeng, J. Hao, S.P. Lau. "Deep Ultraviolet Photoluminescence of Water-Soluble Self-Passivated Graphene Quantum Dots". ACS Nano. 2012. 6(6): 5102-5110.

28. H. Peng, J. Travas-Sejdic. "Simple Aqueous Solution Route to Luminescent Carbogenic Dots from Carbohydrates". Chem. Mater. 2009. 21(23): 5563-5565

29. S.K. Doorn, R.E. Fields III, H. Hu, M.A. Hamon, R.C. Haddon, J.P. Selegue, V. Majidi. "High Resolution Capillary Electrophoresis of Carbon Nanotubes”. J. Am. Chem. Soc. 2002. 124(12): 3169-3174.

30. G. Socrates. Infrared Characteristic Group Frequencies. Chichester, UK: Wiley, 1980.

31. N.B. Colthup, L.H. Daly, S.E. Wiberley. Introduction to Infrared and Raman Spectroscopy. San Diego, CA: Academic Press, 1990. 3rd ed.

32. J.B. Lambert, H.F. Shurvell, D.A. Lightner, R.G. Cooks. Organic Structural Spectroscopy. Upper Saddle River, NJ: Prentice Hall, 1998. 2nd. ed.

33. J. Gulyás, E. Földes, A. Lázár, B. Pukánszky. "Electrochemical Oxidation of Carbon Fibres: Surface Chemistry and Adhesion". Composites A. 2001. 32(3-4): 353-360.

34. P. Taddei, C. Arosio, P. Monti, M. Tsukada, T. Arai, G. Freddi. "Chemical and Physical Properties of Sulfated Silk Fabrics". Biomacromolecules. 2007. 8(4): 1200-1208.

35. W. Kwon, S. Do, S.-W. Rhee. "Formation of Highly Luminescent Nearly Monodisperse Carbon Quantum Dots via Emulsion-Templated Carbonization of Carbohydrates". RSC Adv. 2012. 2(30): 11223-11226. 
36. F.M. Collette, C. Lorentz, G. Gebel, F. Thominette. "Hygrothermal Aging of Nafion". J. Membrane Sci. 2009. 330(1-2): 21-29.

37. V. Lee, L. Whittaker, C. Jaye, K.M. Baroudi, D.A. Fischer, S Banerjee. "Large-Area Chemically Modified Graphene Films: Electrophoretic Deposition and Characterization by Soft X-ray Absorption Spectroscopy". Chem. Mater. 2009. 21(16): 3905-3916.

38. M. Zhang, L. Bai, W. Shang, W. Xie, H. Ma, Y. Fu, D. Fang, H. Sun, L. Fan, M. Han, C. Liu, S. Yang. "Facile Synthesis of Water-Soluble, Highly Fluorescent Graphene Quantum Dots as a Robust Biologica Label for Stem Cells”. J. Mater. Chem. 2012. 22(15): 7461-7467.
39. R. Saito, M. Hofmann, G. Dresselhaus, A. Jorio, M.S. Dresselhaus. "Raman Spectroscopy of Graphene and Carbon Nanotubes". Adv. Phys. 2011. 60(3): 413-550.

40. Y. Liu, C.-Y. Liu, Z.-Y. Zhang. "Synthesis and Surface Photochemistry of Graphitized Carbon Quantum Dots". J. Colloid Interface Sci. 2011. 356(2): 416-421.

41. G. Wang, X. Sun, C. Liu, J. Lian. "Tailoring Oxidation Degrees of Graphene Oxide by Simple Chemical Reactions”. Appl. Phys. Lett. 2011. 99(5): 0531141. 